\title{
OPEN The relationship
}

\section{between specialized pro-resolving lipid mediators, morbid obesity and weight loss after bariatric surgery}

Fabian Schulte ${ }^{1,2,7}$, Abdul Aziz Asbeutah ${ }^{3,6,7}$, Peter N. Benotti ${ }^{4}$, G. Craig Wood ${ }^{4}$, Christopher Still ${ }^{4}$, Bruce R. Bistrian ${ }^{5}$, Markus Hardt ${ }^{1,2}$ \& Francine K. Welty ${ }^{3 凶}$

Obesity and diabetes are associated with chronic inflammation. Specialized pro-resolving lipid mediators (SPMs)-resolvins (Rv), protectins (PD) and maresins (MaR)-actively resolve inflammation. Bariatric surgery achieves remission of diabetes, but mechanisms are unclear. We measured SPMs and proinflammatory eicosanoid levels using liquid chromatography-tandem mass spectrometry in 29 morbidly obese subjects ( 13 with diabetes) and 15 nondiabetic, mildly obese subjects. Compared to the mildly obese, the morbidly obese had higher levels of SPMs-RvD3, RvD4 and PD1-and white blood cells (WBC) and platelets. Post-surgery, SPM and platelet levels decreased in morbidly obese nondiabetic subjects but not in diabetic subjects, suggesting continued inflammation. Despite similar weight reductions 1 year after surgery ( $44.6 \%$ vs. $46.6 \%)$, 8 diabetes remitters had significant reductions in WBC and platelet counts whereas five non-remitters did not. Remitters had a $58.2 \%$ decrease ( $p=0.03$ ) in 14-HDHA, a maresin pathway marker; non-remitters had an $875.7 \%$ increase in 14-HDHA but a 36.9\% decrease in MaR1 to a median of 0 . In conclusion, higher levels of RvD3, PD1 and their pathway marker, 17-HDHA, are markers of leukocyte activation and inflammation in morbid obesity and diabetes and diminish with weight loss in nondiabetic but not diabetic subjects, possibly representing sustained inflammation in the latter. Lack of diabetes remission after surgically-induced weight loss may be associated with reduced ability to produce MaR1 and sustained inflammation.

Visceral adiposity is the major risk factor responsible for the development of insulin resistance common to metabolic syndrome and type 2 diabetes ${ }^{1}$. White adipose tissue releases more than 50 different adipocytokines including the cytokines, tumor necrosis factor (TNF)- $\alpha$ and interleukin (IL)- 6 . TNF- $\alpha$ signaling activates intracellular kinases, c-Jun N-terminal kinase and IкB kinase, leading to increased serine phosphorylation of insulin receptor substrate-1 (IRS-1) which impairs insulin signaling ${ }^{2,3}$, causing insulin resistance that inhibits the transport of glucose into cells $s^{4,5}$.

Inflammation is a protective host response which protects against tissue invasion and injury and initiates healing to minimize tissue damage and restore normal function ${ }^{6}$. In the initiation phase, elevated free fatty acids activate the proinflammatory pathway by upregulating cyclooxygenase (COX) which converts arachidonic acid to proinflammatory, prothrombotic and vasoactive eicosanoids that include prostaglandin $[P G] \mathrm{D}_{2}$, $\mathrm{PGE}_{2}, \mathrm{PGF}_{2 \alpha}$, thromboxane $[\mathrm{Tx}] \mathrm{A}_{2}$ and $\mathrm{TxB}_{2}$, and via 5-lipoxygenase (LOX) which converts arachidonic acid to the pro-inflammatory leukotriene (LT) $\mathrm{B}_{4}$, a potent chemoattractant that recruits neutrophils into tissue to remove necrotic debris and apoptotic cells ${ }^{7,8}$. Neutrophils release TNF- $\alpha$ and IL- 6 which cause insulin resistance

\footnotetext{
${ }^{1}$ Forsyth Institute, Cambridge, MA, USA. ${ }^{2}$ Department of Developmental Biology, Harvard School of Dental Medicine, Boston, MA, USA. ${ }^{3}$ Division of Cardiology, Beth Israel Deaconess Medical Center, Harvard Medical School, 330 Brookline Avenue, SL 423, Boston, MA 02215, USA. ${ }^{4}$ Geisinger Obesity Institute, Danville, PA, USA. ${ }^{5}$ Department of Medicine, Beth Israel Deaconess Medical Center, Harvard Medical School, Boston, MA, USA. ${ }^{6}$ Present address: Department of Medicine, University of Tennessee Health Science Center, Memphis, TN, USA. ${ }^{7}$ These authors contributed equally: Fabian Schulte and Abdul Aziz Asbeutah. ${ }^{\square}$ email: fwelty@ bidmc.harvard.edu
} 
directly via serine phosphorylation of IRS-1. Second, neutrophils upregulate COX and LOX enzymes, which increase production of $\mathrm{LTB}_{4}{ }^{9,10}$, leading to recruitment of more neutrophils, thus prolonging exposure to elevated cytokine levels and furthering insulin resistance. In the resolution phase, production of $\mathrm{PGE}_{2}$ stimulates synthesis of specialized pro-resolving lipid mediators (SPMs) - lipoxins (LX), resolvins (Rv) of the E and D series, protectins (PD) and maresins (MaR) ${ }^{11-13}$. SPMs stop further neutrophil recruitment and stimulate infiltration of monocytes which differentiate into resolution macrophages that phagocytize and clear apoptotic neutrophils and debris via lymphatics, a process called efferocytosis, which is a key step in resolution and prevention of chronic inflammation ${ }^{11,13,14}$.

To date, bariatric surgery is the most effective modality to achieve remission of diabetes, but the mechanisms beyond weight loss are unclear. Our aims were to explore potential mechanisms by examining how SPM and proinflammatory eicosanoid levels change with surgical weight loss.

\section{Methods}

Participants. Subjects with morbid obesity who had lost $>40 \%$ of body weight at 1 year after Roux-enY gastric bypass (RYGB) were identified from the Geisinger prospective Bariatric Surgery biobank ${ }^{15}$. A control group comprised mildly obese subjects without diabetes who had stable clinical CAD. The protocol was approved by the Institutional Review Boards of the Geisinger Clinic and Beth Israel Deaconess Medical Center.

Levels of lipid mediators: sample extraction. Serum samples stored at $-80{ }^{\circ} \mathrm{C}$ were used. Samples were extracted using protein precipitation with methanol followed by solid phase extraction (SPE) using C18 columns as described previously ${ }^{16-19}$. Before extraction, $500 \mathrm{pg}$ of deuterium (d)-labeled internal standards, d5-MaR1, d4-RvE1, d8-5S-hydroxy-eicosatetraenoic acid (d8-5S-HETE), d4-LTB 4 , d5-lipoxin A (d5-LXA $_{4}$ ), $\mathrm{d} 5$-maresin 2 (d5-MaR2), d4-PGE 2 and d5-resolvin D2 (d5-RvD2), were added to facilitate quantification of analyte recovery. Then ten volumes of methanol were added, and the samples stored in the freezer at $-80^{\circ} \mathrm{C}$ for $1 \mathrm{~h}$. After that, the precipitates were centrifuged for $10 \mathrm{~min}$ at $2500 \mathrm{~g}$. Sample supernatants were concentrated to $1 \mathrm{ml}$ in an evaporation station (TurboVap LV, Biotage) as previously described ${ }^{19}$. The evaporated samples were diluted with ten volumes of $\mathrm{ddH} 2 \mathrm{O}$, acidified $(\mathrm{pH} \sim 3.5)$ and loaded onto the $\mathrm{C} 18 \mathrm{SPE}$ column. Before elution, the $\mathrm{C} 18$ resin was washed with $6 \mathrm{ml}$ of neutral ddH2O and $6 \mathrm{ml}$ hexane. Samples were eluted with 6 ml methyl formate and taken to dryness using a nitrogen stream as previously described ${ }^{16-19}$. The extracts were suspended in methanol/water for liquid chromatography-tandem mass spectrometry (LC-MS/MS) analyses.

Liquid chromatography-tandem mass spectrometry analysis. Targeted metabololipidomics using LC-MS/MS was performed to quantify levels of the pro-resolving SPMs (D-series resolvins, E-series resolvins, protectins and maresins) and the proinflammatory eicosanoids $\left(\mathrm{PGD}_{2}, \mathrm{PGE}_{2}\right.$ and $\left.\mathrm{LTB}_{4}\right)$ as previously described $^{16-19}$ using a QTrap 6500 mass spectrometer (Sciex) equipped with a Shimadzu Nexera XR HPLC system. An Agilent Poroshell 120 EC-C18 column $(100 \mathrm{~mm} \times 4.6 \mathrm{~mm} \times 2.7 \mu \mathrm{m})$ was used with aradient of metha$\mathrm{nol} /$ water/acetic acid of 50:50:0.01 (volume/volume/volume) to 100:0:0.01 at 0.5-ml/min flow rate. A scheduled multiple reaction monitoring (MRM) method was used to monitor and quantify the levels of each lipid mediator as we previously described ${ }^{19}$. The MRM detected signature ion fragments for each molecule at chromatographic retention times established by a combination of external and internal synthetic standards as previously described (Supplementary Fig. S1 and Supplementary Table S1 online) ${ }^{19}$. Product ion spectra were collected to confirm the identity of each individual analyte by matching the data with the profiles of at least six published fragment ions as previously described ${ }^{16-19}$. Calibration curves were obtained using synthetic and authentic lipid mediator mixtures. Linear calibration curves for each analyte were obtained at $0.1,1,10$, and $100 \mathrm{pg}$ with $\mathrm{r}^{2}$ values between 0.98 and 0.99 . The peak areas of the SPMs were absolutely quantified based on the linear calibration curves and the recovery rates of the internal standards. The coefficients of variation were $\mathrm{LTB}_{4}: 6.2 \%, \mathrm{MaR} 1: 6.5 \%, \mathrm{RvE} 1$ : $11.2 \%, \mathrm{PGD}_{2}: 13.1 \%$ and $\mathrm{PGE}_{2}: 10 \%$. Reproducibility between laboratories and a methodological validation have been reported ${ }^{20}$.

Statistical analysis. Statistical analyses were performed as previously described ${ }^{21}$. Normality tests were conducted using the Shapiro-Wilk test. Categorical variables were expressed as counts and percentages and compared with either Chi-square or Fisher's exact tests. Continuous variables were reported as the mean and standard deviation (SD) for normally distributed variables or median and interquartile range [IQR] for nonnormally distributed variables. Continuous variables were compared using unpaired Student's t-tests for normally distributed variables or the Mann-Whitney-U test for non-normally distributed variables. A 2-sided $p$ value $\leq 0.05$ was considered statistically significant. Data analyses were performed using SPSS 20.0 for Windows (IBM Corp. Armonk, NY, USA).

Ethical approval. All procedures performed in studies involving human participants were in accordance with the ethical standards of the institutional and/or national research committee and with the 1964 Helsinki declaration and its later amendments or comparable ethical standards.

Informed consent. Informed consent was obtained from all individual participants included in the study. 


\begin{tabular}{|c|c|c|c|}
\hline & Mild obesity $(n=15)$ & Morbid obesity $(n=29)$ & $p$ value \\
\hline \multicolumn{4}{|l|}{ Demographic characteristics } \\
\hline Age (years) & $63.1 \pm 5.4$ & $47.1 \pm 11.0$ & $<0.001$ \\
\hline Female sex, $n(\%)$ & $1(2.3)$ & $27(61.4)$ & $<0.001$ \\
\hline Diabetes mellitus, $n(\%)$ & $0(0 \%)$ & $13(45 \%)$ & \\
\hline \multicolumn{4}{|c|}{ Anthropometric and blood pressure } \\
\hline Weight (kg) & $89.3 \pm 13.6$ & $145.9 \pm 21.4$ & $<0.001$ \\
\hline Weight (lb) & $196.9 \pm 30.1$ & $321.5 \pm 47.1$ & $<0.001$ \\
\hline Waist circumference $(\mathrm{cm})$ & $106.4 \pm 7.7$ & $144.2 \pm 12.4$ & $<0.001$ \\
\hline Waist circumference (inches) & $41.9 \pm 3.0$ & $56.8 \pm 4.9$ & $<0.001$ \\
\hline BMI $\left(\mathrm{kg} / \mathrm{m}^{2}\right)$ & $30.5 \pm 2.7$ & $53.0 \pm 7.3$ & $<0.001$ \\
\hline Systolic BP (mm Hg) & $125.6 \pm 16.1$ & $128.5 \pm 12.7$ & 0.52 \\
\hline Diastolic BP (mm Hg) & $74.2 \pm 9.9$ & $76.0 \pm 8.8$ & 0.55 \\
\hline \multicolumn{4}{|l|}{ Biochemical profile } \\
\hline Glucose (mg/dl) & $94.0 \pm 8.9$ & $111.7 \pm 35.5$ & 0.07 \\
\hline HbAlc (\%) & $5.8 \pm 0.3$ & $6.3 \pm 1.1$ & 0.07 \\
\hline Insulin $(\mathrm{mIU} / \mathrm{ml})$ & $13.3 \pm 8.1$ & $26.8 \pm 14.9$ & 0.002 \\
\hline AST (IU/l) & $22.1 \pm 7.0$ & $28.8 \pm 19.1$ & 0.20 \\
\hline ALT (IU/l) & $25.8 \pm 11.8$ & $30.1 \pm 17.1$ & 0.38 \\
\hline WBC $\left(10^{9}\right.$ cells $\left./ \mathrm{l}\right)$ & $6.07 \pm 1.53$ & $8.51 \pm 2.86$ & 0.004 \\
\hline Platelet count (cells/ $\mu \mathrm{l})$ & $167.2 \pm 40.2$ & $253.14 \pm 86.65$ & 0.001 \\
\hline \multicolumn{4}{|l|}{ Lipids (mg/dl) } \\
\hline Total cholesterol & $154.5 \pm 30.7$ & $185.3 \pm 41.5$ & 0.15 \\
\hline Triglycerides, median [IQR] & $125.0[71.0,188.0]$ & $162.0[148.0,196.5]$ & 0.028 \\
\hline HDL-C & $46.3 \pm 12.3$ & $43.4 \pm 11.4$ & 0.44 \\
\hline LDL-C & $80.1 \pm 23.2$ & $105.1 \pm 32.2$ & $<0.001$ \\
\hline TC/HDL ratio & $3.6 \pm 1.4$ & $4.4 \pm 1.1$ & 0.028 \\
\hline
\end{tabular}

Table 1. Baseline characteristics for mildly obese nondiabetic subjects compared to morbidly obese subjects. ALT Alanine transaminase, AST aspartate transaminase, BMI body mass index, $H b A 1 C$ hemoglobin A1c, $H D L-C$ high density lipoprotein, $L D L-C$ low density lipoprotein-calculated, $T C$ total cholesterol, $W B C$ white blood cells. Continuous variables are presented as mean \pm SD or median [interquartile range] and categorical variables are presented as $n(\%)$. A 2 -sided $p$ value $\leq 0.05$ was considered statistically significant.

\section{Results}

Baseline characteristics of subjects. The Geisinger Clinic subjects consisted of 29 subjects with morbid obesity (mean body mass index $[\mathrm{BMI}] \pm$ SD: $53.0 \pm 7.3 \mathrm{~kg} / \mathrm{m}^{2}$ ) who had lost $>40 \%$ of their initial weight at 1 year post-bariatric surgery. Compared to 15 mildly obese (mean BMI: $30.5 \pm 2.7 \mathrm{~kg} / \mathrm{m}^{2}$ ) nondiabetic subjects, the morbidly obese subjects were significantly younger, more likely to be female and had significantly higher BMI, waist circumference and levels of insulin, triglyceride and LDL-C, presumably reflecting their greater level of obesity and associated metabolic derangements (Table 1). They also had significantly higher levels of white blood cell count (WBC) and platelets, indicating a higher level of inflammation. The morbidly obese also had a borderline significantly higher level of $\mathrm{PGE}_{2}$, a proinflammatory mediator, which is a signal to switch to the proresolving phase and bring in SPMs-PD1, RvD3 and RvD4-to resolve inflammation.

Baseline SPM and proinflammatory eicosanoid levels before surgery. Table 2 reports the lowest calibration standard on the calibration curve (LOQ) and the limit of detection (LOD) for our mass spectrometry measurements. Figure 1 reports the intraday extraction and detection reproducibility for SPMs isolated from independent blood samples $(n=5)$. Before surgery, compared to the 15 mildly obese nondiabetic subjects, the morbidly obese subjects had significantly higher levels of 14-HDHA (a marker of the maresin [MaR1] pathway) and the DHA-derived SPMs, PD1, resolvin D3 (RvD3) and RvD4, and $\mathrm{PGE}_{2}$ and significantly lower levels of 17-HDHA, a marker of the pathway for both RvD3 and PD1 and precursor for RvD3 (Table 3).

Baseline characteristics in morbidly obese subjects stratified by diabetes status. Of the 29 morbidly obese subjects, 13 had type 2 diabetes. Before surgery, compared to the nondiabetic subjects, the diabetic subjects had significantly higher levels of glucose, $95 \pm 11 \mathrm{mg} / \mathrm{dl} \mathrm{vs} 131 \pm 45 \mathrm{mg} / \mathrm{dl}$, respectively, $p=0.02$, and hemoglobin (Hb) A1c, $5.6 \% \pm 0.4$ vs $7.1 \% \pm 1.2$, respectively, $p=0.001$ (Table 4 ).

SPM and proinflammatory eicosanoid levels in obese diabetic and nondiabetic subjects before and after surgery. Table 5 reports no significant differences in weight, BMI, WBC or platelet count before or after surgery in the morbidly obese nondiabetic subjects compared to the diabetic subjects. Both nondiabetic 


\begin{tabular}{|l|l|l|}
\hline Compound & LOD $^{\mathbf{a}}$ (pg per injection) & LOQ $^{\mathbf{b}}$ (pg per injection) \\
\hline $\mathrm{d} 4-\mathrm{LTB}_{4}$ & 0.5 & 1.0 \\
\hline $\mathrm{d} 4-\mathrm{PGE}_{2}$ & 0.5 & 1.0 \\
\hline $\mathrm{d} 5-\mathrm{LXA}_{4}$ & 0.1 & 0.2 \\
\hline $\mathrm{d} 5-\mathrm{MaR} 2$ & 1.0 & 2.0 \\
\hline $\mathrm{d} 5-\mathrm{RvD} 2$ & 0.1 & 0.2 \\
\hline $\mathrm{d} 8-5-\mathrm{HETE}$ & 0.1 & 0.1 \\
\hline 14-HDHA & 0.1 & 0.1 \\
\hline 17-HDHA & 0.1 & 0.2 \\
\hline 18-HEPE & 0.5 & 1.0 \\
\hline LTB $_{4}$ & 0.5 & 1.0 \\
\hline $\mathrm{LXA}_{4}$ & 0.1 & 0.2 \\
\hline $\mathrm{LXB}_{4}$ & 0.1 & 0.2 \\
\hline MaR1 & 0.2 & 0.5 \\
\hline MaR2 & 0.5 & 1.0 \\
\hline PD1 & 0.1 & 0.5 \\
\hline PDX & 0.1 & 0.2 \\
\hline PGD & 0.1 & 0.1 \\
\hline PGE & 0.1 & 0.1 \\
\hline RvD1 & 0.2 & 0.5 \\
\hline RvD2 & 0.2 & 0.5 \\
\hline RvD3 & 0.1 & 0.2 \\
\hline RvD4 & 0.1 & 0.1 \\
\hline RvD5 & 0.1 & 0.1 \\
\hline RvE1 & 0.2 & 0.5 \\
\hline & & \\
\hline
\end{tabular}

Table 2. Intraday extraction and detection reproducibility for SPMs isolated from independent blood samples $(\mathrm{n}=5)$. $d$ deuterium, $L T B_{4}$ leukotriene $\mathrm{B}_{4}, L X A_{4}$ lipoxin $\mathrm{A}_{4}, L X B_{4}$ lipoxin $\mathrm{B}_{4}, M a R 1$ maresin $1, M a R 2$ maresin 2, $P D 1$ protectin $\mathrm{D} 1, P D X$ protectin $\mathrm{X}, P G D_{2}$ prostaglandin $\mathrm{D}_{2}, P G E_{2}$ prostaglandin $\mathrm{E}_{2}, R v D 1$ resolvin $\mathrm{D} 1$, $R v D 2$ resolvin $\mathrm{D} 2, R v D 3$ resolvin $\mathrm{D} 3, R v D 4$ resolvin $\mathrm{D} 4, R v D 5$ resolvin $\mathrm{D} 5, R v E 1$ resolvin $\mathrm{E} 1, S P M$ specialized pro-resolving lipid mediators, 14-HDHA 14-hydroxy-docosahexaenoic acid, 17-HDHA 17-hydroxydocosahexaenoic acid, 18-HEPE 18-hydroxy-eicosapentaenoic acid. ${ }^{\mathrm{a}}$ Limit of detection. ${ }^{\mathrm{b}}$ Limit of quantitation.

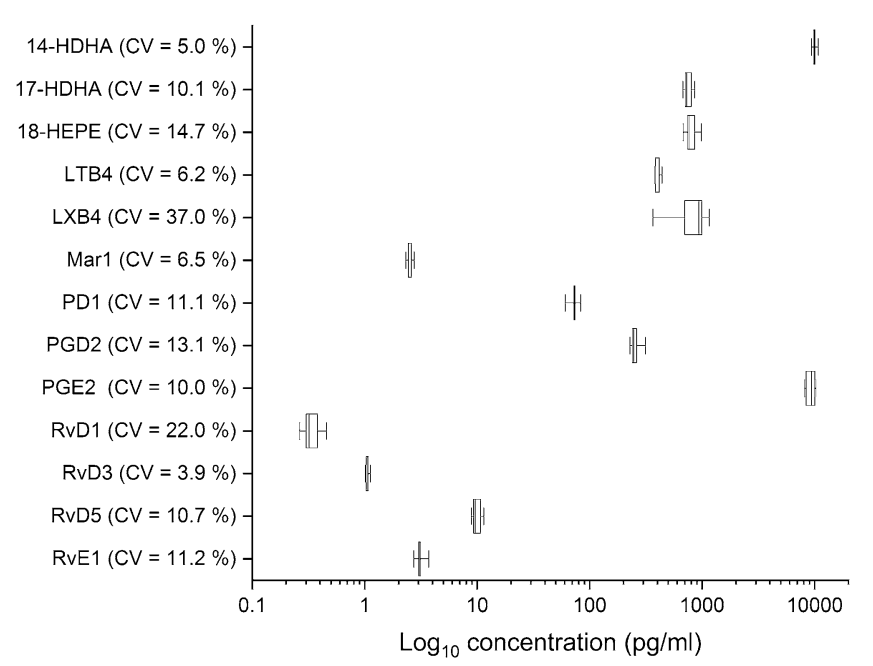

Figure 1. Intraday extraction and detection reproducibility for SPMs isolated from independent blood samples $(\mathrm{n}=5)$. Data expressed as median [interquartile range] and whiskers showing the minimum and maximum values. $L T B_{4}$ leukotriene $\mathrm{B}_{4}, L X B_{4}$ lipoxin $\mathrm{B}_{4}, M a R 1$ maresin $1, P D 1$ protectin $\mathrm{D} 1, P G D_{2}$ prostaglandin $\mathrm{D}_{2}$, $P G E_{2}$ prostaglandin $\mathrm{E}_{2}, R v D 1$ resolvin $\mathrm{D} 1, R v D 3$ resolvin $\mathrm{D} 3, R v D 5$ resolvin $\mathrm{D} 5, R v E 1$ resolvin $\mathrm{E} 1, S P M$ specialized pro-resolving lipid mediators, 14-HDHA 14-hydroxy-docosahexaenoic acid, 17-HDHA 17-hydroxydocosahexaenoic acid, 18-HEPE 18-hydroxy-eicosapentaenoic acid. 


\begin{tabular}{|l|l|l|c|}
\hline SPM $(\mathbf{\rho g} / \mathbf{m l})$ & Mild obesity $(\boldsymbol{n}=\mathbf{1 5})$ & Morbid obesity $(\boldsymbol{n}=\mathbf{2 9})$ & $\boldsymbol{p}$ value \\
\hline $14-\mathrm{HDHA}$ & $36.9[22.4,289.6]$ & $483.4[170.7,749.1]$ & 0.011 \\
\hline $17-\mathrm{HDHA}$ & $152.0[118.1,298.5]$ & $90.8[72.1,139.0]$ & $<0.001$ \\
\hline $18-\mathrm{HEPE}$ & $67.5[42.1,79.0]$ & $40.7[27.5,66.7]$ & 0.040 \\
\hline $\mathrm{LTB}_{4}$ & $32.5[19.2,76.9]$ & $19.6[0,72.3]$ & 0.34 \\
\hline $\mathrm{LXA}_{4}$ & $0[0,0]$ & $0[0,0.14]$ & 0.13 \\
\hline $\mathrm{LXB}_{4}$ & $0[0,0]$ & $0[0,2.0]$ & 0.34 \\
\hline MaR1 & $24.1[0,41.3]$ & $9.1[0,25.3]$ & 0.69 \\
\hline MaR 2 & $0[0,0]$ & $0[0,0]$ & 0.14 \\
\hline PD1 & $0[0,0]$ & $67.6[55.9,83.7]$ & $<0.001$ \\
\hline PDX & $0[0,0]$ & $0[0,0]$ & 0.40 \\
\hline PGD & $4.9[3.6,14.8]$ & $4.7[2.9,9.1]$ & 0.21 \\
\hline PGE 2 & $1.7[0,27.6]$ & $14.5[2.6,49.7]$ & 0.054 \\
\hline RvD1 & $8.1[0,17.4]$ & $5.1[3.5,8.8]$ & 0.15 \\
\hline RvD2 & $0[0,0]$ & $0[0,0]$ & 0.63 \\
\hline RvD3 & $0.6[0,2.2]$ & $2.4[1.7,2.9]$ & 0.036 \\
\hline RvD4 & $0[0,0]$ & $241.3[144.3,389.6]$ & $<0.001$ \\
\hline RvD5 & $0[0,0]$ & $0[0,0]$ & 0.16 \\
\hline RvE1 & $0[0,0]$ & $0[0,0]$ & 0.38 \\
\hline
\end{tabular}

Table 3. Lipid mediators in mildly obese nondiabetic subjects compared to morbidly obese subjects adjusted for age. $L T B_{4}$ leukotriene $\mathrm{B}_{4}, L X A_{4}$ lipoxin $\mathrm{A}_{4}, L X B_{4}$ lipoxin $\mathrm{B}_{4}, M a R 1$ maresin $1, M a R 2$ maresin 2, PD1 protectin $\mathrm{D} 1, P D X$ protectin $\mathrm{X}, P G D_{2}$ prostaglandin $\mathrm{D}_{2}, P G E_{2}$ prostaglandin $\mathrm{E}_{2}, R v D 1$ resolvin $\mathrm{D} 1, R v D 2$ resolvin $\mathrm{D} 2, R v D 3$ resolvin $\mathrm{D} 3, R v D 4$ resolvin $\mathrm{D} 4, R v D 5$ resolvin $\mathrm{D} 5, R v E 1$ resolvin $\mathrm{E} 1, S P M$ specialized pro-resolving lipid mediators, 14-HDHA 14-hydroxy-docosahexaenoic acid, 17-HDHA 17-hydroxydocosahexaenoic acid, 18-HEPE 18-hydroxy-eicosapentaenoic acid.

\begin{tabular}{|c|c|c|c|}
\hline & No diabetes $(n=16)$ & Diabetes $(n=13)$ & $p$ value \\
\hline \multicolumn{4}{|l|}{ Demographic characteristics } \\
\hline Age (years) & $45.50 \pm 8.60$ & $49.08 \pm 13.49$ & 0.39 \\
\hline Female sex $n(\%)$ & $14(87.5)$ & $13(100)$ & 0.19 \\
\hline \multicolumn{4}{|c|}{ Anthropometric and blood pressure } \\
\hline Weight $(\mathrm{kg})$ & $143.0 \pm 20.0$ & $134.4 \pm 22.9$ & 0.67 \\
\hline Weight (lb) & $314.5 \pm 46.0$ & $295.7 \pm 43.3$ & 0.97 \\
\hline Waist circumference $(\mathrm{cm})$ & $146.2 \pm 13.5$ & $141.6 \pm 10.8$ & 0.32 \\
\hline Waist circumference (inches) & $57.6 \pm 5.3$ & $55.8 \pm 4.3$ & 0.32 \\
\hline BMI $\left(\mathrm{kg} / \mathrm{m}^{2}\right)$ & $53.3 \pm 7.6$ & $52.7 \pm 7.2$ & 0.84 \\
\hline Systolic BP (mm Hg) & $128.3 \pm 10.1$ & $128.8 \pm 15.7$ & 0.92 \\
\hline Diastolic BP (mm Hg) & $76.3 \pm 9.1$ & $75.5 \pm 8.8$ & 0.82 \\
\hline \multicolumn{4}{|l|}{ Biochemical profile } \\
\hline Glucose (mg/dl) & $95.9 \pm 10.8$ & $131.2 \pm 45.3$ & 0.02 \\
\hline HbAlc (\%) & $5.6 \pm 0.4$ & $7.1 \pm 1.2$ & 0.001 \\
\hline Insulin $(\mathrm{mIU} / \mathrm{ml})$ & $29.8 \pm 16.2$ & $22.7 \pm 12.4$ & 0.22 \\
\hline AST (IU/l) & $25.1 \pm 9.5$ & $33.3 \pm 26.4$ & 0.26 \\
\hline ALT (IU/l) & $28.9 \pm 12.6$ & $31.7 \pm 21.9$ & 0.67 \\
\hline \multicolumn{4}{|l|}{ Lipids (mg/dl) } \\
\hline Total Cholesterol & $190.0 \pm 45.1$ & $179.6 \pm 37.5$ & 0.51 \\
\hline Triglycerides, median [IQR] & $162.50[152.0,190.8]$ & $162.00[127.5,208.5]$ & 0.92 \\
\hline HDL-C & $44.1 \pm 10.0$ & $42.7 \pm 13.4$ & 0.75 \\
\hline LDL-C & $110.3 \pm 36.2$ & $98.8 \pm 26.5$ & 0.35 \\
\hline TC/HDL ratio & $4.4 \pm 0.8$ & $4.5 \pm 1.4$ & 0.68 \\
\hline
\end{tabular}

Table 4. Baseline characteristics for morbidly obese nondiabetic subjects compared to morbidly obese diabetic subjects. ALT Alanine transaminase, AST aspartate transaminase, BMI body mass index, $H b A 1 C$ hemoglobin A1c, $H D L-C$ high density lipoprotein, $L D L$-C low density lipoprotein, $T C$ total cholesterol. Continuous variables are presented as mean \pm SD or median [interquartile range] and categorical variables are presented as $n(\%)$. A 2 -sided $p$ value $\leq 0.05$ was considered statistically significant. 


\begin{tabular}{|c|c|c|c|c|c|c|c|c|}
\hline & \multicolumn{3}{|c|}{ Nondiabetic subjects $(n=16)$} & \multicolumn{5}{|c|}{ Diabetic subjects $(n=13)$} \\
\hline & Pre-surgery & Post-surgery & $p^{\mathrm{a}}$ & Pre-surgery & Post-surgery & $p^{\mathrm{b}}$ & $p^{c}$ & $p^{\mathrm{d}}$ \\
\hline Wt (lb) & $314.5 \pm 46.0$ & $178.0 \pm 27.3$ & $<0.001$ & $295.7 \pm 43.3$ & $170.6 \pm 26.2$ & $<0.001$ & 0.97 & 0.76 \\
\hline $\mathrm{BMI}^{\mathrm{e}}$ & $53.3 \pm 7.6$ & $28.8 \pm 4.6$ & $<0.001$ & $52.7 \pm 7.2$ & $28.9 \pm 4.3$ & $<0.001$ & 0.77 & 0.58 \\
\hline $\mathrm{WBC}^{\mathrm{f}}$ & $8.41 \pm 2.29$ & $5.74 \pm 2.08$ & $<0.001$ & $8.64 \pm 3.54$ & $6.39 \pm 1.75$ & 0.007 & 0.46 & 0.58 \\
\hline Platelets $^{\mathrm{g}}$ & $252.1 \pm 81.2$ & $195.1 \pm 75.0$ & $<0.001$ & $254.5 \pm 96.3$ & $232.2 \pm 125.9$ & 0.10 & 0.82 & 0.49 \\
\hline \multicolumn{9}{|c|}{\begin{tabular}{|l} 
Docosahexaenoic acid metabolome $(\rho \mathrm{g} / \mathrm{ml})$ \\
\end{tabular}} \\
\hline RvD1 & $4.8[3.9,8.5]$ & $4.3[3.4,5.7]$ & 0.33 & $5.4[3.1,8.9]$ & $6.1[5,2,12.3]$ & 0.22 & \begin{tabular}{|l|}
0.78 \\
\end{tabular} & 0.045 \\
\hline RvD3 & $2.5[1.8,3.2]$ & $1.5[1.3,2.3]$ & 0.026 & $2.1[1.6,2.7]$ & $1.6[1.5,2.4]$ & 0.60 & 0.42 & 0.45 \\
\hline RvD4 & $245.7[145.5,432.8]$ & $179.9[123.6,426.1]$ & 0.38 & $228.0[141.1,366.7]$ & $188.8[125.2,370.8]$ & 0.55 & 0.62 & 0.98 \\
\hline 17 HDHA & $99.9[77.9,153.5]$ & $69.1[39.5,133.8]$ & 0.026 & $88.1[57.8,124.0]$ & $56.2[42.6,116.7]$ & 0.60 & 0.37 & 0.95 \\
\hline PD1 & $68.9[58.0,83.9]$ & $43.2[39.6,70.3]$ & 0.002 & $64.2[47.9,83.7]$ & $59.1[39.9,75.9]$ & 0.65 & 0.56 & 0.50 \\
\hline 14-HDHA & $558.7[213.5,806.2]$ & $345.7[203.4,845.2]$ & 0.50 & $254.0[56.2,708.1]$ & $401.1[126.3,942.9]$ & 0.65 & 0.22 & 0.85 \\
\hline Mar1 & $9.5[0,34.2]$ & $30.0[1.3,47.7]$ & 0.43 & $8.8[0,21.2]$ & $0[0,6.2]$ & 0.21 & 0.81 & 0.012 \\
\hline \multicolumn{9}{|c|}{ Eicosapentaenoic acid metabolomes $(\rho \mathrm{g} / \mathrm{ml})$} \\
\hline RvE1 & $0[0,0]$ & $0[0,0]$ & 0.32 & $0[0,0]$ & $0[0,0]$ & 1.00 & 0.78 & 1.00 \\
\hline 18-HEPE & $44.0[26.6,67.9]$ & $31.2[20.9,53.4]$ & 0.070 & $36.2[24.4,61.2]$ & $34.6[20.0,61.2]$ & 0.38 & 0.56 & 0.98 \\
\hline \multicolumn{9}{|c|}{ Arachidonic acid metabolomes ( $\mathrm{\rho g} / \mathrm{ml})$} \\
\hline $\mathrm{LTB}_{4}$ & $29.0[0,109.5]$ & $22.4[2.0,57.2]$ & 0.18 & $15.2[0,38.8]$ & $0[0,43.0]$ & 1.00 & 0.48 & 0.25 \\
\hline $\mathrm{PGD}_{2}$ & $6.3[3.1,8.2]$ & $6.0[3.2,8.8]$ & 0.20 & $4.1[2.5,9.6]$ & $4.6[2.7,8.2]$ & 0.70 & 0.62 & 0.56 \\
\hline $\mathrm{PGE}_{2}$ & $19.0[5.1,70.9]$ & $37.0[14.0,84.0]$ & 0.72 & $13.8[0,39.5]$ & $16.9[9.4,29.6]$ & 0.81 & 0.29 & 0.11 \\
\hline
\end{tabular}

Table 5. Characteristics and SPM and eicosanoid levels in morbidly obese nondiabetic subjects compared to diabetic subjects before and after bariatric surgery. $B M I$ Body mass index, $L T B_{4}$ leukotriene $\mathrm{B}_{4}, M a R 1$ maresin $1, P D 1$ protectin D1, $\rho g$ picogram, $P G D_{2}$ prostaglandin $\mathrm{D}_{2}, P G E_{2}$ prostaglandin $\mathrm{E}_{2}, R v D 1$ resolvin $\mathrm{D} 1$, $R v D 3$ resolvin $\mathrm{D} 3, R v D 4$ resolvin $\mathrm{D} 4, R v E 1$ resolvin $\mathrm{E} 1$, Wt weight, $14-H D H A$ 14-hydroxy-docosahexaenoic acid, 17-HDHA 17-hydroxy-docosahexaenoic acid, 18-HEPE 18-hydroxy-eicosapentaenoic acid. Continuous variables are presented as mean $\pm \mathrm{SD}$ or median [interquartile range]. A 2 -sided $p$ value $\leq 0.05$ was considered statistically significant. ${ }^{\mathrm{a}} p$ value comparing nondiabetic subjects pre-surgery to post-surgery. ${ }^{\mathrm{b}} p$ value comparing diabetic subjects pre-surgery to post-surgery. ${ }^{c} p$ value comparing nondiabetic subjects to diabetic subjects pre-surgery. ${ }^{\mathrm{d}} p$ value comparing nondiabetic subjects to diabetic subjects post-surgery. ${ }^{\mathrm{e}} \mathrm{BMI}$ expressed as $\mathrm{kg} / \mathrm{m}^{2}$. ${ }^{\mathrm{f}} \mathrm{WBC}$ expressed as $10^{9}$ cells $/ \mathrm{l}$. ${ }^{\mathrm{g}}$ Platelets expressed as cells $/ \mu \mathrm{l}$.

and diabetic subjects had similar significant reductions in weight ( $>44 \%$ of total body weight) and BMI after surgery. WBC decreased significantly in both nondiabetic and diabetic subjects after bariatric surgery; however, platelet count decreased significantly only in nondiabetic subjects. Both WBC and platelet count are markers of inflammation and the lack of a significant reduction in platelet count in the diabetic subjects may indicate a higher level of residual inflammation post-surgery. No significant differences were observed in the proinflammatory eicosanoids and pro-resolving SPMs between the morbidly obese nondiabetic subjects and diabetic subjects before surgery, presumably reflecting higher inflammation due to excess weight in both groups. After surgery, RvD3 and PD1 and their pathway marker, 17-HDHA, were significantly lower in nondiabetic subjects compared to values before surgery, consistent with decreased inflammation. In contrast, these SPMs did not change in the diabetic subjects after surgery, presumably indicating failure to resolve inflammation despite similar weight loss. Of note, RvD1 was significantly higher and MaR1 was significantly lower in the diabetic subjects compared to nondiabetic subjects after surgery ( $p=0.045$ and 0.012 , respectively).

SPM and proinflammatory eicosanoid levels before and after bariatric surgery stratified by diabetes remission. Diabetes remission was defined as being off diabetic medications and an HbAlc $<6.5 \%{ }^{22,23}$. Eight diabetic subjects had remission whereas five did not. Table 6 reports that both groups had similar reductions in weight, $44.6 \%$ vs. $46.6 \%$ and BMI, $44.8 \%$ vs. $44.9 \%$. After surgery, the remitters had significant reductions in levels of WBC and platelet count whereas the non-remitters did not, indicating a higher level of inflammation in non-remitters. Compared to those who did not remit, those who remitted had significantly higher 14-HDHA levels $(p=0.019)$ before surgery, and they continued to produce the active SPM, MaR1, from its precursor 14-HpDHA after surgery. After surgery, non-remitters had an $875.7 \%$ increase in 14-HDHA, (from 46 to $454 \rho \mathrm{g} / \mathrm{ml}$ with the $\%$ increase significant, $p=0.021)$ whereas remitters had a $58.2 \%$ decrease $(p=0.030)$. The non-remitters had a $36.9 \%$ decrease in MaR1 to a median value of 0 , suggesting that 14 -HpDHA is preferentially converted to 14-HDHA rather than to MaR1 in the non-remitters. The mean ratio of 14-HDHA/MaR1 was significantly different in remitters compared to non-remitters both pre- and post-surgery $(p=0.019$ and 0.016 , respectively) (Table 6). Thus, it appears that there is a relative impairment in the ability to produce MaR1, the active SPM, in non-remitters which may impact the anti-inflammatory, pro-resolving actions of SPMs. 


\begin{tabular}{|c|c|c|c|c|c|c|c|c|c|c|c|}
\hline & \multicolumn{3}{|l|}{ Pre-surgery } & \multicolumn{3}{|l|}{ Post-surgery } & \multicolumn{5}{|c|}{ Pre vs. Post } \\
\hline & Remitters $(n=8)$ & $\begin{array}{l}\text { Non-remitters } \\
(n=5)\end{array}$ & $p$ & Remitters $(n=8)$ & $\begin{array}{l}\text { Non-remitters } \\
(n=5)\end{array}$ & $p$ & $p^{\mathrm{a}}$ & $p^{\mathrm{b}}$ & $\begin{array}{l}\% \text { change in } \\
\text { remitters }\end{array}$ & $\begin{array}{l}\% \text { change in } \\
\text { nonremitters }\end{array}$ & $p^{c}$ \\
\hline Weight (lb) & $290.0 \pm 43.8$ & $304.9 \pm 45.6$ & 0.57 & $167.6 \pm 26.2$ & $175.2 \pm 28.4$ & 0.63 & $<0.001$ & $<0.001$ & $-44.6 \pm 6.2$ & $-46.6 \pm 4.2$ & 0.55 \\
\hline $\mathrm{BMI}^{\mathrm{d}}$ & $51.0 \pm 7.6$ & $55.3 \pm 6.5$ & 0.32 & $28.0 \pm 4.2$ & $30.5 \pm 4.4$ & 0.32 & $<0.001$ & $<0.001$ & $-44.8 \pm 7.2$ & $-44.9 \pm 3.3$ & \begin{tabular}{|l|l|}
0.97 \\
\end{tabular} \\
\hline $\mathrm{WBC}^{\mathrm{e}}$ & $8.62 \pm 1.77$ & $8.68 \pm 5.67$ & 0.97 & $6.00 \pm 0.93$ & $6.99 \pm 2.63$ & 0.35 & 0.004 & 0.35 & $\begin{array}{l}-30.1[-43.4, \\
-15.9]\end{array}$ & $\begin{array}{l}-13.4[-39.3, \\
25.1]\end{array}$ & 0.58 \\
\hline Platelets $^{\mathrm{f}}$ & $234 \pm 28$ & $287 \pm 156$ & 0.36 & $195 \pm 25$ & $292 \pm 198$ & 0.19 & 0.016 & 0.83 & $\begin{array}{l}-21.6[-23.8, \\
-6.3]\end{array}$ & $\begin{array}{l}-3.2[-17.8, \\
13.2]\end{array}$ & 0.062 \\
\hline $14 \mathrm{HDHA}$ & $\begin{array}{l}339.1[246.2, \\
1076.4]\end{array}$ & $46.5[33.1,324.7]$ & 0.019 & $\begin{array}{l}\text { 153.9 [109.6, } \\
988.7]\end{array}$ & \begin{tabular}{|l}
$454.1[317.6$, \\
$1012.0]$
\end{tabular} & 0.28 & 0.58 & 0.14 & $\begin{array}{l}-58.2[-86.7, \\
118.5]\end{array}$ & $\begin{array}{l}+875.7[225.1 \\
3076.4]\end{array}$ & 0.030 \\
\hline MaR1 & $9.6[0,23.0]$ & $8.8[0,32.6]$ & 0.94 & $1.8[0,16.6]$ & $0[0,2.8]$ & 0.35 & 0.75 & 0.11 & $0[-80.8,10.4]$ & $\begin{array}{l}-36.9[-100.0, \\
0]\end{array}$ & 0.35 \\
\hline 14 HDHA/MaR1 & $51.6 \pm 93.4$ & $1.7 \pm 2.1$ & 0.019 & $11.1 \pm 14.9$ & $45.5 \pm 101.6$ & 0.016 & 0.29 & 0.39 & & & \\
\hline 17 HDHA & $\begin{array}{l}\text { 103.2 [71.4, } \\
148.6]\end{array}$ & $81.6[24.5,91.5]$ & 0.17 & $49.2[40.7,116.6]$ & $99.3[45.8,116.7]$ & 0.62 & 0.26 & 0.35 & $\begin{array}{l}-52.7[-67.9, \\
53.4]\end{array}$ & $\begin{array}{l}+45.85[-27.6 \\
509.2]\end{array}$ & 0.19 \\
\hline $18 \mathrm{HEPE}$ & $37.2[31.4,49.9]$ & $33.4[15.6,105.7]$ & 0.94 & $29.9[19.9,46.0]$ & $35.1[17.7,81.6]$ & 0.72 & 0.48 & 0.69 & $\begin{array}{l}-10.7[-63.0, \\
42.8]\end{array}$ & $\begin{array}{l}-46.5[-62.9, \\
318.8]\end{array}$ & 0.83 \\
\hline $\mathrm{LTB}_{4}$ & $21.5[14.0,40.1]$ & $0[0,89.3]$ & 0.17 & $12.1[0,43.1]$ & $0[0,67.2]$ & 0.72 & 0.89 & 1.00 & $\begin{array}{l}+100.0[-100.0, \\
23.8]\end{array}$ & $+497.5[-100.0]^{\mathrm{g}}$ & 0.89 \\
\hline $\mathrm{LXB}_{4}$ & $0[0,0]$ & $0[0,2.2]$ & 0.35 & $0.51[0,2.7]$ & $2.3[0,3.6]$ & 0.44 & 0.068 & 0.47 & & & \\
\hline PD1 & $61.7[47.7,84.3]$ & $67.6[48.8,83.7]$ & 0.94 & $64.0[39.4,81.5]$ & $52.9[38.0,71.5]$ & 0.62 & 0.89 & 0.23 & $\begin{array}{l}+7.6[-30.0, \\
41.2]\end{array}$ & $\begin{array}{l}-24.8[-36.5, \\
6.2]\end{array}$ & 0.35 \\
\hline $\mathrm{PGD}_{2}$ & $3.2[2.3,8.3]$ & $8.7[3.1,10.9]$ & 0.44 & $3.2[2.5,13.4]$ & $4.8[3.8,8.2]$ & 0.52 & 0.16 & 0.50 & $\begin{array}{l}+36.1[-12.2, \\
57.8]\end{array}$ & $\begin{array}{l}-37.6[-51.4, \\
109.6]\end{array}$ & 0.44 \\
\hline $\mathrm{PGE}_{2}$ & $18.8[3.4,55.4]$ & $3.5[0,23.8]$ & 0.22 & $17.2[7.4,30.1]$ & $16.9[10.5,111.1]$ & 0.72 & 0.31 & 0.14 & $\begin{array}{l}-36.4[-66.3, \\
14.8]\end{array}$ & $+378.0[-22.2]^{\mathrm{g}}$ & 0.048 \\
\hline RvD1 & $5.3[2.8,9.3]$ & $6.6[4.0,8.9]$ & 0.83 & $7.8[5.1,12.7]$ & $6.1[4.6,10.8]$ & 1.00 & 0.40 & 0.35 & $\begin{array}{l}+80.5[-34.8, \\
331.7]\end{array}$ & $\begin{array}{l}+22.1[-6.8, \\
35.4]\end{array}$ & 0.44 \\
\hline RvD3 & $1.8[1.5,2.6]$ & $2.5[1.8,3.0]$ & 0.35 & $1.7[1.4,2.3]$ & $1.6[1.3,3.1]$ & 0.94 & 0.67 & 0.69 & $\begin{array}{l}+5.8[-16.7, \\
17.0]\end{array}$ & $\begin{array}{l}+5.1[-50.5, \\
16.2]\end{array}$ & 0.83 \\
\hline RvD4 & $\begin{array}{l}221.0[141.2, \\
330.5]\end{array}$ & $\begin{array}{l}343.7[74.1, \\
473.2]\end{array}$ & 0.62 & $\begin{array}{l}187.9[105.8, \\
382.5]\end{array}$ & $\begin{array}{l}314.3[150.2, \\
372.5]\end{array}$ & 0.72 & 0.67 & 0.50 & $\begin{array}{l}-11.6[-33.7, \\
73.1]\end{array}$ & $\begin{array}{l}-9.3[-31.1, \\
-1.5]\end{array}$ & 0.81 \\
\hline
\end{tabular}

Table 6. Pre- and post-surgery characteristics and SPM and eicosanoid levels in diabetic subjects with remission of diabetes (remitters) compared to diabetic subjects without remission of diabetes (non-remitters) after bariatric surgery. $B M I$ Body mass index, $L T B_{4}$ leukotriene $\mathrm{B}_{4}, L X A_{4}$ lipoxin $\mathrm{A}_{4}, L X B_{4}$ lipoxin $\mathrm{B}_{4}, M a R 1$ maresin $1, P D 1$ protectin $\mathrm{D} 1, P G D_{2}$ prostaglandin $\mathrm{D}_{2}, P G E_{2}$ prostaglandin $\mathrm{E}_{2}, R v D 1$ resolvin $\mathrm{D} 1, R v D 3$ resolvin $\mathrm{D} 3, R v D 4$ resolvin $\mathrm{D} 4, W B C$ white blood cells 14-HDHA 14-hydroxy-docosahexaenoic acid, 17-HDHA 17-hydroxy-docosahexaenoic acid, 18-HEPE 18-hydroxy-eicosapentaenoic acid. Continuous variables are presented as mean \pm SD or median [interquartile range]. A 2 -sided $p$ value $\leq 0.05$ was considered statistically significant. ${ }^{a} p$ value comparing pre- to post-surgery values in remitters using Wilcoxon signed Rank test. ${ }^{\mathrm{b}} p$ value comparing pre- to post-surgery values in non-remitters using Wilcoxon signed Rank test. ${ }^{c} p$ value comparing \% change in remitters to non-remitters. ${ }^{\mathrm{d}} \mathrm{BMI}$ expressed as $\mathrm{kg} / \mathrm{m}^{2}$. ${ }^{\mathrm{e}} \mathrm{WBC}$ expressed as $10^{9}$ cells $/ \mathrm{l}$. fPlatelets expressed as cells/ $\mu \mathrm{l} .{ }^{\mathrm{g}} 75$ th quartile could not be calculated due to only three evaluable individuals.

\section{Discussion}

In the current study, we examined the effect of morbid obesity and the effect of $>40 \%$ weight loss after bariatric surgery on levels of proinflammatory eicosanoids and pro-resolving SPMs in nondiabetic and diabetic subjects. We report several new findings. First, before surgery, the morbidly obese subjects had significantly higher levels of the DHA-derived, pro-resolving mediators, PD1, RvD3 and RvD4, compared to mildly obese subjects, presumably reflecting a compensatory response to higher inflammation in the morbidly obese. The morbidly obese also had a borderline significantly higher level of $\mathrm{PGE}_{2}$, a proinflammatory mediator, which is a signal to switch to the pro-resolving phase and bring in SPMs-PD1, RvD3 and RvD4 - to resolve inflammation. In contrast, no significant difference was observed in the proinflammatory eicosanoids and pro-resolving SPMs in morbidly obese diabetic subjects compared to morbidly obese nondiabetic subjects before surgery, presumably due to excess weight in both groups.

A second major finding is that after surgery, the nondiabetic subjects had a significant decrease in the $\mathrm{D}$-series resolvins, RvD3 and PD1, and their pathway marker, 17-HDHA, compared to pre-surgery, presumably indicating an improvement in the inflammatory state post-surgery in the nondiabetic subjects Thus, the SPM levels indicate the state of inflammation: high pre-surgery in morbid obesity with and without diabetes reflecting inflammation and diminishing SPM levels post weight loss in the nondiabetic subjects, a finding indicating a reduction in inflammation. In 197 subjects undergoing sleeve gastrectomy with $B M I \geq 35 \mathrm{~kg} / \mathrm{m}^{2}$ pre-surgery, the classical inflammatory marker, high-sensitivity C-reactive protein (hs-CRP) decreased from $8.8 \mathrm{mg} / \mathrm{l}(0.6-45.4)$ pre-surgery to $2.6 \mathrm{mg} / 1(0.1-126.1)$ at 12 months after surgery $(p<0.001)^{24}$; therefore, the decrease in SPMs postsurgery in the current study also reflects a reduction in inflammation. 
In contrast, RvD3, PD1 and 17-HDHA levels did not decrease in the diabetic subjects after surgery. This finding suggests that diabetic subjects may maintain higher levels of SPMs reflecting a continued elevated inflammatory stress due to diabetes, a proinflammatory state with elevated free fatty acid levels and continued insulin resistance. The decrease in WBC after bariatric surgery suggests a decrease in inflammation in both nondiabetic and diabetic groups as does the decrease in platelet count in the nondiabetic subjects. However, the lack of change in platelet count in the diabetic subjects is consistent with residual inflammation consistent with the unaltered SPM levels. Systemic inflammation as manifested by elevated levels of hs-CRP has been demonstrated in obesity and in type 2 diabetes ${ }^{24-30}$. WBC and platelet counts are also markers of inflammation and both were elevated in the morbidly obese compared to the mildly obese in the current study. Total WBC count has predicted CAD incidence and mortality in patients without CAD and predicted mortality in patients with $\mathrm{CAD}^{31}$. In a metaanalysis of 5337 subjects without CAD in 7 large studies, a high baseline WBC count was associated with a 1.4fold increased risk of CAD (95\% CI 1.3-1.5), comparable to that of hs-CRP $(1.45,95 \% \text { CI } 1.25-1.68)^{32,33}$. The higher WBC and platelet counts along with higher SPM levels in the morbidly obese compared to the mildly obese in the current report support an increased level of inflammation as a compensatory response to on-going inflammation.

A third major finding is the relationship between SPMs and diabetes remission. DHA is converted to 14-HpDHA which in turn is converted to either 14-HDHA or MaR1. Before surgery, those with remission at 1 year had tenfold higher 14-HDHA levels than non-remitters and they maintained levels of the active SPM, MaR1, after surgery. In contrast, post-surgery, the non-remitters had a tenfold increase in 14-HDHA levels; however, the median value for MaR1 fell to 0, indicating a block in the conversion of the precursor, 14-HpDHA, to MaR1 in the non-remitters. The median value for MaR1 was also 0 in the total group of diabetic subjects after surgery and significantly lower compared to the nondiabetic subjects. Perhaps higher pre-operative SPM levels of 14-HDHA indicate higher pro-resolution capacity which might predict the ability to remit.

A prior study, the Longitudinal Assessment of Bariatric Surgery cohort, examined serum biomarkers of inflammation and adiposity as predictors of diabetes remission after bariatric surgery. In 2458 subjects, hs-CRP levels decreased more after RYBG compared to gastric banding ${ }^{34}$. However, neither hs-CRP nor ghrelin, leptin or cystatin $\mathrm{C}$ correlated with metabolic disease remission after surgery in either group. Therefore, baseline biomarkers did not predict disease remission. In the STAMPEDE trial, 150 diabetic subjects were randomized to intensive medical treatment alone, RYBG or sleeve gastrectomy. At 5-year follow-up after bariatric surgery, weight loss was $23.2 \pm 9.6 \mathrm{~kg}$ after RYGB and $18.6 \pm 7.5 \mathrm{~kg}$ after sleeve gastrectomy with a mean decrease in hs-CRP of $75 \%$ after RYGB and $69 \%$ after sleeve gastrectomy ${ }^{35}$. Remission of diabetes defined as not taking diabetic medications was $51 \%$ after RYGB and $25 \%$ after sleeve gastrectomy. These studies suggest that the level of inflammation falls after bariatric surgery due to weight loss, but there appears to be no relation between hs-CRP level and diabetes remission. In contrast, the current findings suggest that SPMs may be predictive of remission of diabetes and should be examined further in future studies.

To our knowledge, the current study is the first to report on serum levels of pro-resolving SPMs and proinflammatory eicosanoids in morbidly obese patients before and after surgically-induced weight loss and the impact of coexisting diabetes. The results suggest that SPMs may not only be a marker of inflammation but may also track the likelihood of diabetes remission. Support for this notion is found in animal models of obesity and diabetes which suggest that defective macrophage efferocytosis and apoptotic cell uptake due to free fatty acids and obesity can be reversed by the addition of SPMs. In obese diabetic mice, elevated free fatty acids prolonged neutrophil survival at a phase when neutrophils normally undergo apoptosis and are cleared from the inflammatory site ${ }^{36}$. Prolonged neutrophil survival leads to an increase in insulin resistance. Inflamed visceral and subcutaneous fat compartments from ob/ob obese mice, $\mathrm{db} / \mathrm{db}$ obese/diabetic mice and high-fat diet-induced obese mice demonstrated deficits in RvD1 and PD1 and their pathway marker, $17 \mathrm{HDHA}^{37,38}$. Consistent with this notion that defective SPM biosynthesis promotes adipose tissue inflammation, the administration of nanogram doses of $\mathrm{RvD} 1$ to $\mathrm{db} / \mathrm{db}$ obese diabetic mice resulted in an improvement in glucose tolerance, a reduction in fasting blood glucose, an increase in insulin-stimulated Akt phosphorylation in adipose tissue and a reduction in the crown-like structures rich in inflammatory macrophages in adipose tissue ${ }^{39}$. Similarly, injection of nanogram amounts of RvE1 in the peritoneum of obese ob/ob mice demonstrated a significant insulin-sensitizing effect ${ }^{38}$. Moreover, injection of 17-HDHA, the precursor of the D-series resolvins, in the peritoneum of $\mathrm{db} / \mathrm{db}$ obese diabetic mice showed a reduction in adipose tissue expression of inflammatory cytokines (MCP-1, TNF- $\alpha$ and IL-6), an increase in the expression of the anti-inflammatory adiponectin and an improvement in glucose tolerance which was associated with increased insulin sensitivity ${ }^{38}$. A lower ratio of SPM to $\mathrm{LTB}_{4}$ and SPM to PGs has been confirmed in omental white adipose tissue obtained from obese patients undergoing bariatric surgery ${ }^{40}$, a finding extending the deficit observed in animal models to the human. This imbalance may lead to an inadequate tissue resolution capacity and allow the inflammatory response to continue. Administration of RvD1 increased the IL-10 induced anti-inflammatory response and promoted the secretion of the anti-inflammatory cytokine, IL-10, in obese diabetic mice ${ }^{39,41}$, thus highlighting the pro-resolving functions of these lipid mediators.

Diet may play an important role as well. Compared to mice receiving a standard chow diet, aging mice ( $\geq 18$ months old) receiving a diet enriched in omega-6 fatty acids (safflower oil-linoleic acid) had higher levels of the proinflammatory mediators, $\mathrm{LTB}_{4}$ and $\mathrm{TxB}_{2}$, and cardiac cytokines, both indicating low-grade chronic inflammation ${ }^{42}$. They also had increased nighttime carbohydrate utilization and atrio-ventricular block on dobutamine-induced stress test, findings suggesting an adverse effect of enrichment in dietary omega- 6 fatty acids. When subjected to ligature of the coronary artery to cause myocardial infarction (MI), despite similar infarct size and left ventricular dysfunction post-MI, those on the safflower oil diet had decreased 5-, 12- and 15-lipoxygenases in the infarcted left ventricle, lower levels of pro-resolving mediators and increased expression of TNF- $\alpha$ and IL- $1 \beta$ compared to mice receiving the chow diet ${ }^{43}$. In contrast, feeding an omega- 3 enriched diet to obese $\mathrm{ob} / \mathrm{ob}$ mice resulted in increased levels of the pro-resolving mediators, RvD1, PD1 and their pathway marker, 
17-HDHA, in visceral white adipose tissue ${ }^{37}$. Nanomolar concentrations of LXA 4 decreased IL-6 and restored the glucose transporter, GLUT4, and IRS-1 in adipose explants from aging female mice, a finding indicating reduced inflammation and improved insulin sensitivity ${ }^{44}$. $\mathrm{LXA}_{4}$ has also been shown to preserve AKt signaling and glucose uptake in cultured adipocytes ${ }^{44}$. Transgenic restoration of the SPM precursors, eicosapentaenoic acid (EPA) and docosahexaenoic acid (DHA), reversed the loss of resolution capacity in adipose tissue from fat1 obese mice, a finding suggesting the activity of the pro-resolution SPM pathways depends on how much EPA and DHA are consumed in the diet ${ }^{45}$. These findings suggest that the failure of adipose tissue to produce SPMs to resolve inflammation can contribute to obesity-linked inflammation and insulin resistance. Altogether, these and our present findings strongly suggest a potential role for SPMs in obesity-linked inflammation and insulin resistance and that dietary EPA and DHA can influence the pro-resolution SPM pathways. Whether increasing SPMs in severe obesity in humans by dietary increase in EPA and DHA would favorably reduce inflammation and thereby have some clinical benefit particularly in diabetes will require further clinical study.

In conclusion, the major findings in the current study are: (1) higher levels of SPMs, WBC and platelet count in morbidly obese subjects compared to mildly obese subjects indicate a higher level of inflammation in morbidly obese subjects; (2) the decrease in SPMs in nondiabetic but not in diabetic subjects after surgery with $>40 \%$ weight loss in both is consistent with a greater reduction in inflammation in nondiabetic subjects; the lack of reduction in diabetic subjects suggests that diabetes promotes continued inflammation; and (3) the lack of remission of diabetes after surgically-induced weight loss is associated with a reduced ability to produce a specific SPM, namely MaR1. Future studies are needed to determine if SPMs are only a marker of inflammation or perhaps are, or can be, actively involved in resolving the inflammatory process in severe obesity.

\section{Data availability}

All data generated or analyzed during this study are included in the published article (and its "Supplementary Information" file).

Received: 16 April 2020; Accepted: 22 September 2020

Published online: 18 November 2020

\section{References}

1. Welty, F. K., Alfaddagh, A. \& Elajami, T. K. Targeting inflammation in metabolic syndrome. Transl. Res. 167, 257-280 (2016).

2. Gao, Z. et al. Serine phosphorylation of insulin receptor substrate 1 by inhibitor $\kappa$ B kinase complex. J. Biol. Chem. 277, 48115-48121 (2002).

3. Zhang, J., Gao, Z., Yin, J., Quon, M. J. \& Ye, J. S6K directly phosphorylates IRS-1 on Ser-270 to promote insulin resistance in response to TNF-a signaling through IKK2. J. Biol. Chem. 283, 35375-35382 (2008).

4. Donath, M. Y. \& Shoelson, S. E. Type 2 diabetes as an inflammatory disease. Nat. Rev. Immunol. 11, $98-107$ (2011).

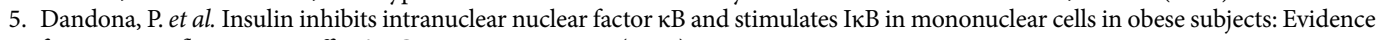
for an anti-inflammatory effect?. JCEM. 86, 3257-3265 (2001).

6. Kumar, V., Abbas, A. K. \& Aster, J. C. Inflammation and repair. In Robbins and Cotran Pathologic Basis of Disease 9th edn (eds Kumar, V. et al.) 69-111 (Elsevier/Saunders, Philadelphia, 2015).

7. Samuelsson, B. Role of basic science in the development of new medicines: Examples from the eicosanoid field. J. Biol. Chem. 287, $10070-10080$ (2012).

8. Haeggström, J. Z. \& Funk, C. D. Lipoxygenase and leukotriene pathways: Biochemistry, biology, and roles in disease. Chem. Rev. 111, 5866-5898 (2011).

9. Kasama, T. et al. Neutrophil-derived cytokines: Potential therapeutic targets in inflammation. Curr. Drug Targets Inflamm. Allergy. 4, 273-279 (2005).

10. Scher, J. U., Abramson, S. B. \& Pillinger, M. H. Neutrophils I. In Fundamentals of Inflammation (eds Serhan, C. N. et al.) (Cambridge University Press, Cambridge, 2010).

11. Serhan, C. N. Pro-resolving lipid mediators are leads for resolution physiology. Nature 510, 92-101 (2014).

12. Levy, B. D., Clish, C. B., Schmidt, B., Gronert, K. \& Serhan, C. N. Lipid mediator class switching during acute inflammation: Signals in resolution. Nat. Immunol. 2, 612-619 (2001).

13. Spite, M., Clària, J. \& Serhan, C. N. Resolvins, specialized proresolving lipid mediators, and their potential roles in metabolic diseases. Cell Metab. 19, 21-36 (2014).

14. Tabas, I. \& Glass, C. K. Anti-Inflammatory therapy in chronic disease: Challenges and opportunities. Science 339, 166-172 (2013).

15. Wood, G. C. et al. An electronic health record-enabled obesity database. BMC Med Dec. Mak. Inform. 12, 45. https://doi. org/10.1186/1472-6947-12-45 (2012).

16. Colas, R. A., Shinohara, M., Dalli, J., Chiang, N. \& Serhan, C. N. Identification and signature profiles for pro-resolving and inflammatory lipid mediators in human tissue. Am. J. Physiol. Cell Physiol. 307, C39-54. https://doi.org/10.1152/ajpcell.00024.2014 (2014).

17. Yang, R., Chiang, N., Oh, S. F. \& Serhan, C. N. Metabolomics-lipidomics of eicosanoids and docosanoids generated by phagocytes. Curr. Protoc. Immunol. 95, 1-26. https://doi.org/10.1002/0471142735.im1426s95 (2011).

18. Hong, S. et al. Resolvin D1, protectin D1, and related docosahexaenoic acid-derived products: Analysis via electrospray/low energy tandem mass spectrometry based on spectra and fragmentation mechanisms. J. Am. Soc. Mass. Spectrom. 18, 128-144 (2007).

19. Parashar, K., Schulte, F., Hardt, M. \& Baker, O. J. Sex-mediated elevation of the specialized pro-resolving lipid mediator levels in a Sjögren's syndrome mouse model. FASEB J. https://doi.org/10.1096/fi.201902196R (2020).

20. Norris, P. C. et al. Identification of specialized pro-resolving mediator clusters from healthy adults after intravenous low-dose endotoxin and omega-3 supplementation: A methodological validation. Sci. Rep. 8, 18050. https://doi.org/10.1038/s41598-01836679-4 (2018).

21. Al Faddagh, A. et al. An omega-3 fatty acid plasma index $\geq 4 \%$ prevents progression of coronary artery plaque in patients with coronary artery disease on statin treatment. 285, 153-162 (2019). https://doi.org/10.1016/j.atherosclerosis.2019.04.213.

22. Buse, J. B. et al. How do we define cure of diabetes?. Diabetes Care 32, 2133-2135 (2009).

23. Still, C. D. et al. A probability score for preoperative prediction of type 2 diabetes remission following RYGB surgery. Lancet Diabetes Endocrinol. 2, 38-45 (2014).

24. Randell, E. W. et al. Pre-operative and post-operative changes in CRP and other biomarkers sensitive to inflammatory status in patients with severe obesity undergoing laparoscopic sleeve gastrectomy. Clin. Biochem. 52, 13-19 (2018).

25. Visser, M., Bouter, L. M., McQuillan, G. M., Wener, N. H. \& Harris, T. B. Elevated C-reactive protein levels in overweight and obese adults. JAMA 282, 2131-2135 (1999). 
26. Ebrahimi, M. et al. Association of serum hs-CRP levels with the presence of obesity, diabetes mellitus, and other cardiovascular risk factors. J. Clin. Lab. Anal. 30, 672-676 (2016).

27. Arismendi, E. et al. The systemic inflammome of severe obesity before and after bariatric surgery. PLoS ONE 9, e107859. https:// doi.org/10.1371/journal.pone.0107859 (2014).

28. Chiappetta, S. et al. The impact of obesity and metabolic surgery on chronic inflammation. Obes. Surg. 28, 3028-3040 (2018).

29. Paepegaey, A. C. et al. High levels of CRP in morbid obesity: The central role of adipose tissue and lessons for clinical practice before and after bariatric surgery. Surg. Obes. Relat. Dis. 11, 148-154 (2015).

30. Careaga, M. et al. Inflammation and iron status in bariatric surgery candidates. Surg. Obes. Relat. Dis. 11, 906-911 (2015).

31. Madjid, M. \& Fatemi, O. Components of the complete blood count as risk predictors for coronary heart disease: In-depth review and update. Tex. Heart Inst. J. 40, 17-29 (2013).

32. Danesh, J. et al. C-reactive protein and other circulating markers of inflammation in the prediction of coronary heart disease. $N$. Engl. J. Med. 350, 1387-1397 (2004).

33. Danesh, J., Collins, R., Appleby, P. \& Peto, R. Association of fibrinogen, C-reactive protein, albumin, or leukocyte count with coronary heart disease: Meta-analyses of prospective studies. JAMA 279, 1477-1482 (1998).

34. O'Rourke, R. W. et al. Serum biomarkers of inflammation and adiposity in the LABS cohort: Associations with metabolic disease and surgical outcomes. Int. J. Obes. 43, 285-296 (2018).

35. Bhatt, D. L. et al. Cardiovascular biomarkers after metabolic surgery versus medical therapy for diabetes. J. Am. Coll. Cardiol. 74, 261-263 (2019).

36. Bannenberg, G. L. et al. Molecular circuits of resolution: Formation and actions of resolvins and protectins. J. Immunol. 174, 4345-4355 (2005).

37. González-Périz, A. et al. Obesity-induced insulin resistance and hepatic steatosis are alleviated by $\omega$-3 fatty acids: A role for resolvins and protectins. FASEB J. 23, 1946-1957 (2009).

38. Neuhofer, A. et al. Impaired local production of proresolving lipid mediators in obesity and 17-HDHA as a potential treatment for obesity-associated inflammation. Diabetes 62, 1945-1956 (2013).

39. Hellmann, J., Tang, Y., Kosuri, M., Bhatnagar, A. \& Spite, M. Resolvin D1 decreases adipose tissue macrophage accumulation and improves insulin sensitivity in obese-diabetic mice. FASEB J. 25, 2399-2407 (2011).

40. Titos, E. et al. Signaling and immunoresolving actions of resolvin D1 in inflamed human visceral adipose tissue. J. Immunol. 197, 3360-3370 (2016).

41. Clària, J., Dalli, J., Yacoubian, S., Gao, F. \& Serhan, C. N. Resolvin D1 and resolvin D2 govern local inflammatory tone in obese fat. J. Immunol. 189, 2597-2605 (2012).

42. Vasundhara, K. et al. Excess $\omega-6$ fatty acids influx in aging drives metabolic dysregulation, electrocardiographic alterations, and low-grade chronic inflammation. Am. J. Physiol. Heart Circ. Physiol. 314, H-160-H-169. https://doi.org/10.1152/ajpheart.00297 2017 (2018)

43. Halade, G. V., Kain, V., Black, L. M., Prabhul, S. D. \& Ingle, K. A. Aging dysregulates D- and E-series resolvings to modulate cardiosplenic and cardiorenal network following myocardial infarction. Aging. 18, 2611-2634 (2016).

44. Börgeson, E. et al. Lipoxin $\mathrm{A}_{4}$ attenuates adipose inflammation. FASEB J. 26, 4287-4294 (2012).

45. White, P. J., Arita, M., Taguchi, R., Kang, J. X. \& Marette, A. Transgenic restoration of long-chain n-3 fatty acids in insulin target tissues improves resolution capacity and alleviates obesity-linked inflammation and insulin resistance in high-fat-fed mice. Diabetes 59, 3066-3073 (2010).

\section{Acknowledgements}

Funding was provided by the Center for Nutritional Research Charitable Trust. We thank the study subjects for participating.

\section{Author contributions}

F.K.W. conceived and designed the experiments. C.S. and C.W. enrolled subjects and C.S. performed bariatric surgery. F.S. and M.H. performed the mass spectrometry analyses. A.A.A. performed statistical analyses. F.K.W., B.R.B. and P.N.B. contributed to critical discussion and data interpretation. F.K.W. supervised the whole process. F.K.W. drafted the manuscript and F.K.W., B.R.B., P.N.B., M.H. and F.S. edited the manuscript. All authors reviewed and approved the final version of the manuscript.

\section{Competing interests}

The authors declare no competing interests.

\section{Additional information}

Supplementary information is available for this paper at https://doi.org/10.1038/s41598-020-75353-6.

Correspondence and requests for materials should be addressed to F.K.W.

Reprints and permissions information is available at www.nature.com/reprints.

Publisher's note Springer Nature remains neutral with regard to jurisdictional claims in published maps and institutional affiliations.

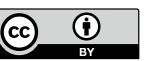

Open Access This article is licensed under a Creative Commons Attribution 4.0 International License, which permits use, sharing, adaptation, distribution and reproduction in any medium or format, as long as you give appropriate credit to the original author(s) and the source, provide a link to the Creative Commons licence, and indicate if changes were made. The images or other third party material in this article are included in the article's Creative Commons licence, unless indicated otherwise in a credit line to the material. If material is not included in the article's Creative Commons licence and your intended use is not permitted by statutory regulation or exceeds the permitted use, you will need to obtain permission directly from the copyright holder. To view a copy of this licence, visit http://creativecommons.org/licenses/by/4.0/.

(C) The Author(s) 2020 